\title{
Potential cancer risk with omalizumab: a disproportionality analysis of the WHO's VigiBase pharmacovigilance database
}

\author{
Diogo Mota ${ }^{1}$, Tiago Rama ${ }^{2}$, Milton Severo ${ }^{3}$, and André Moreira ${ }^{1}$ \\ ${ }^{1}$ Faculty of Medicine \\ ${ }^{2}$ Faculdade de Medicina da Universidade do Porto, Porto, Portugal \& Centro Hospitalar \\ São João \\ ${ }^{3}$ Faculty of Medicine, University of Porto
}

April 13, 2021

\section{Potential cancer risk with omalizumab: a disproportionality analysis of the WHO's VigiBase pharmacovigilance database}

To the editor,

An increasing body of evidence strongly suggests that immunoglobulin E (IgE) plays a key role in cancer immune surveillance. Moreover, a possible link between absent or very low serum IgE levels and malignancy risk has also been suggested ${ }^{1}$. Still, two studies on omalizumab, a widely used anti-IgE drug, failed to suggest any influence on the development or progression of malignancy ${ }^{2,3}$. However, they were based on the analysis of data from efficacy clinical trials, including carefully selected participants, enrolled for a limited time, and neither powered nor designed to assess long-term complications such as incident cancer. Therefore, we aimed to examine the incidence of malignancy using comprehensive data from real-life omalizumab-treated patients.

We performed a disproportionality analysis (case/non-case study) ${ }^{4}$ within VigiBase, the World Health Organization's global database of individual case safety reports, to identify a signal of cancer, expressed as the reporting odds-ratio [ROR] and its $95 \%$ confidence interval [CI] for omalizumab. ${ }^{5}$ Cases were defined as Adverse Drug Reactions (ADR) coded as Neoplasms according to the Medical Dictionary for Regulatory Activities (MedDRA) terminology reported between 2000 and 2020. Non-cases were defined as all other ADRs during the same period. The main analysis estimated the risk of reporting "Neoplasms" associated with Omalizumab compared with all other ADRs for Omalizumab to the remaining reported drugs. Analysis for other selected cancers according to MedDRA terminology was also performed.

A total of 1380 reports mentioned neoplasms associated with omalizumab. The disproportionality signal was significant and positive: ROR $[95 \% \mathrm{CI}]=1.65$ [1.56 to 1.74$]$. (Table 1 ). The association was particularly strong in breast and lung cancer, with 232 cases and ROR $[95 \% \mathrm{CI}]=4.12[3.61-4.69]$ and, 85 cases and ROR $[95 \% \mathrm{CI}]=3.04[2.45-3.76]$ respectively (Table 1$)$.

The finding that omalizumab increases by two-thirds the risk of incident cancer is of major relevance but should be interpreted with caution. Disproportionality analysis is exploratory in the context of signal detection, not allowing quantification of the true risk. These studies have been shown to detect early signals that later are confirmed by controlled studies. ${ }^{4}$ As such, a confirmatory study is needed to ascertain our findings. Information present in VigiBase comes from a variety of sources and the probability that the suspected adverse effect is drug-related is not the same in all cases. Additionally, under-reporting may have occurred. However, when referring to serious events, like cancer, this is less prone to happen and, if it did, would 
increase the strength of the signal. Similarly, the notoriety effect cannot apply as former studies failed to show any association between omalizumab and cancer.

Our study has important strengths. Our analysis was based on the most global and comprehensive pharmacovigilance database. Moreover, the safety of omalizumab was evaluated using post-marketing surveillance data overcoming the limitations of clinical trials inclusion criteria and limited follow-up.

In conclusion, using real-world data mining, we reported a signal that omalizumab may be associated with a significantly higher risk of malignancies. Until further studies confirm the long-term safety of omalizumab, this observation should be reflected on the benefit-risk assessment when considering anti-IgE for the treatment of allergic conditions.

\section{References}

1. Ferastraoaru D, Bax HJ, Bergmann C, et al. AllergoOncology: Ultra-low IgE, a potential novel biomarker in cancer - A Position Paper of the European Academy of Allergy and Clinical Immunology (EAACI). Clin Transl Allergy . 2020;10(1):1-16. doi:10.1186/s13601-020-00335-w

2. Busse W, Buhl R, Fernandez Vidaurre C, et al. Omalizumab and the risk of malignancy: results from a pooled analysis. J Allergy Clin Immunol . 2012;129(4):983-989.e6. doi:10.1016/j.jaci.2012.01.033

3. Long A, Rahmaoui A, Rothman KJ, et al. Incidence of malignancy in patients with moderateto-severe asthma treated with or without omalizumab. J Allergy Clin Immunol . 2014;134(3). doi:10.1016/j.jaci.2014.02.007

4. Faillie JL. Case-non-case studies: Principle, methods, bias and interpretation. Therapie . 2019;74(2):225232. doi:10.1016/j.therap.2019.01.006

5. Montastruc JL, Sommet A, Bagheri H, Lapeyre-Mestre M. Benefits and strengths of the disproportionality analysis for identification of adverse drug reactions in a pharmacovigilance database. Br J Clin Pharmacol . 2011;72(6):905-908. doi:10.1111/j.1365-2125.2011.04037.x

Authors:

Diogo Mota ${ }^{1}$

Tiago Azenha Rama ${ }^{1,2}$

Milton Severo ${ }^{3}$

André Moreira ${ }^{1,2,3}$

${ }^{1}$ Serviço de Imunoalergologia, Centro Hospitalar Universitário de São João, Porto, Portugal;

${ }^{2}$ Serviço de Imunologia Básica e Clínica, Faculdade de Medicina da Universidade do Porto, Portugal;

${ }^{3}$ EPIUnit, Instituto de Saúde Pública, Universidade do Porto, e Laboratório para a Investigação Integrativa e Translacional em Saúde Populacional (ITR), Porto, Portugal

\section{Corresponding author:}

André Moreira

andremoreira@med.up.pt

EPIUnit, Instituto de Saúde Pública, Universidade do Porto, e Laboratório para a Investigação Integrativa e Translacional em Saúde Populacional (ITR), Porto, Portugal

\section{Acknowledgments}

The information does not represent the opinion of the Uppsala Monitoring Centre (UMC) or the World Health Organization. 


\section{Declaration of Competing Interest}

All authors disclose no financial and personal relationships with other people or organizations that could inappropriately influence their work.

\section{Contributors}

DM drafted the manuscript. AM conceived, and coordinated subsequent edits and revisions. MS and TR participated in drafting the manuscript and its finalization. All authors have read and approved the final manuscript.

Table 1 Disproportionality analysis (reporting odds ratio [ROR] and its 95\% confidence interval [CI]) of selected cancers and total neoplasms for omalizumab in VigiBase for the period between 2000 and 2020.

\begin{tabular}{lllll}
\hline Neoplasm & Cases associated with Omalizumab (No.) & Total cases, (No.) & ROR & $95 \%$ CI \\
\hline Breast cancer & 232 & 34449 & 4.12 & $3.61-4.69$ \\
Lung cancer & 85 & 17012 & 3.04 & $2.45-3.76$ \\
Prostate cancer & 57 & 13625 & 2.54 & $1.96-3.30$ \\
Colon cancer & 46 & 7595 & 3.68 & $2.76-4.92$ \\
Malignant melanoma & 45 & 8258 & 3.31 & $2.47-4.44$ \\
Thyroid cancer & 22 & 3841 & 3.48 & $2.29-5.30$ \\
Leukemia & 21 & 4604 & 2.77 & $1.80-4.25$ \\
Neoplasms (total) & 1380 & 515120 & 1.65 & $1.56-1.74$ \\
\hline
\end{tabular}

